\title{
Intense Sweeteners, Appetite for the Sweet Taste, and Relationship to Weight Management
}

\author{
France Bellisle
}

Published online: 11 January 2015

(C) Springer Science+Business Media New York 2015

\begin{abstract}
High intensity, low-energy sweeteners (LES) are used by many consumers in order to limit energy intake and possibly facilitate body weight control. These beneficial effects are often questioned in the scientific and lay media. LES are frequently accused of stimulating and/or maintaining a liking for sweetness which in turn would be deleterious for adequate body weight control. Evidence for the specific effects of LES use on appetite for sweet products will be extracted from observational studies, experimental laboratory studies, randomized controlled trials, and finally brain imaging studies. While many of the existing studies cannot identify any causal links between use of LES and appetite for sweetness, randomized trials in children and adults suggest that use of LES tends to reduce rather than increase the intake of sugarcontaining foods and to facilitate, rather than impair, weight loss.
\end{abstract}

Keywords Intense sweeteners · Sweetness · Appetite · Weight control

\section{Introduction}

Sweetness is a potent psychobiological stimulus for many animal species, and particularly for humans of all ages. Sweetness clearly increases the palatability of numerous foods and beverages, and stimulates intake [1]. The potent attraction

This article is part of the Topical Collection on Psychological Issues

F. Bellisle $(\bowtie)$

Université Paris 13, Epidémiologie Nutritionnelle, Centre de

Recherche en Nutrition Humaine d'Ile-de-France, U1153 INSERM,

U1125 INRA, CNAM, 74 rue Marcel Cachin, 93017 Bobigny

cedex, France

e-mail: f.bellisle@uren.smbh.univ-paris13.fr of humans to sweet tasting foods and beverages has given rise to the notion that the appetite for sweet products may stimulate overeating and induce weight gain over the long term. In fact, in traditional food products, sweetness is brought by sugars, carbohydrates with a distinctive sweet taste, which also bring calories: $4 \mathrm{kcal}$ per gram. A high appetite for such energy-rich substances can facilitate overconsumption and, in a society where palatable and convenient foods are widely available, override the physiological energy-regulation mechanisms $[2,3]$. In order to allow consumers to enjoy the palatable sweet taste of their favorite foods and beverages without the energy load of sugar, a number of intense sweetening agents have been developed in the last decades. These substances have a very high sweetening power compared to sugars, so that they can be used in minute amounts to confer the desired level of sweetness to foods and drinks, while contributing very little or no energy at all to the final product.

Over the years, such intense, low-energy sweeteners (LES) have raised concerns and fears. They have been accused of fueling the worldwide obesity epidemic [4], of increasing the risk for diabetes and cardiovascular disease [5], and of inducing metabolic derangements [6]. These concerns have been extensively examined elsewhere [7]. The present review will address different issues. It has been suggested that intense LES enhance the natural appetite for sweetness, exacerbate the liking for and intake of sweet products of all kinds, and prevent consumers from managing their response to sweetness. Such a view was expressed notably in the French Nutrition and Health Program (PNNS), whose website [8] claims that LES products prevent a "weaning" from sweet taste, in the same fashion as weaning occurs from maternal milk in infants or perhaps from a dependence-inducing drug. The notion of "addiction" to palatable foods has indeed been proposed in the scientific literature $[9,10]$ and it is interesting to examine how this concept applies to sugar, LES, or to sweetness itself. 


\section{LES and Body Weight Control}

There is currently much research on the biological mechanisms that drive the consumption of sweet-tasting foods. One key concern is whether prolonged exposure to sweettasting, yet non- or low-energy foods can disrupt the psycho-physiological links between taste responsiveness, appetite, and energy intake. Can exposure to LES enhance the appetite for sweet foods and drinks? Do LES stimulate the intake of sweet tasting products? Do LES affect body weight control?

The notion that LES might enhance appetite and intake is not a new one. It was formulated in the 1980s by John Blundell and his team [11-15]. Blundell underlined that sweetness is a potent psycho-physiological stimulus of appetite and intake. The novel situation created by intense, low-calorie sweeteners is that they uncouple sweet taste and energy content. When a sweet-tasting and energycontaining product is ingested, the sensory stimulation is followed by post-ingestive effects that act to limit intake; such effects include alliesthesia (a decrease in experienced pleasantness of the food) [16] and the metabolic responses to energy-yielding sugars. By contrast, according to Blundell's early hypothesis, while LES stimulate appetite via their sweet taste, they exert no post-ingestive inhibitory influence [11]. The experience of sweetness in the absence of calories, for example when having a diet drink, might weaken the natural sweetness-energy relationship and consequently disrupt appetite control mechanisms. Blundell made it clear that he never claimed that LES per se, and particularly aspartame, stimulated appetite and intake [14]. His view was that the experience of sweetness itself was a stimulus of appetite and ingestion.

In accordance with this hypothesis, Blundell and his group published a number of studies showing an increase in energy intake following the presentation of various foods or beverages sweetened with LES [11-13]. These early results have not been replicated by numerous later works that failed to show any stimulatory effect of LES on appetite and/or energy intake. The vast literature in this field is reviewed periodically and systematic reviews and meta-analyses $[17,18]$ concluded that the use of LES, as opposed to sugar, actually induced a decrease in daily energy intake and facilitated weight loss in dieters.

The present article will not revisit the just cited literature about energy intake and body weight control, but will rather concentrate on studies examining how the use of LES by human consumers might potentially modify the appetite for and the intake of sweet tasting products. A few studies conducted in Europe and America, using very different methodological approaches, shed some light on potential effects.

\section{Observational Studies}

The large-scale epidemiological SuViMax study included 12, 000 French adults, aged 45-60 years at the start of an 8 yearfollow-up of dietary intake and health [19]. In this doubleblind randomized study, one experimental group received daily supplementation with various vitamins and minerals at doses usually found in a healthy diet, while a control group received a placebo. All took part in the same physical examinations and answered the same questionnaires about diet, exercise, and a number of elements of lifestyle. Among the participants about $20 \%$ of males and $30 \%$ of females were users of LES at least occasionally [20]. It was observed that users were slightly but significantly heavier than non-users (about 1.5 BMI point) and had higher fasting levels of glycemia and triacylglycerol. Users of LES also had a lower daily energy intake (about $4 \%$ ) than non-users, and ingested significantly less sugar. According to food choices reported in dietary questionnaires, it appeared that users of LES ingested less potatoes and less sweets, but more yoghurt than nonusers. Overall, these data are consistent with the notion that users of LES are individuals with higher weight control difficulties than non-users, who are actively trying to decrease their intake of sugars via healthy food selection in addition to the use of LES at least occasionally. The results provided no evidence suggesting enhanced appetite in general, or higher appetite for sugar and sweet products in users of LES.

A study of purchases at the supermarket was conducted in a group of 1574 American adults living alone [21]. Examination of cash register receipts showed that the purchase of LES "light" drinks was associated with the purchase of "healthy foods" (fruits and vegetables for example), but not with the purchase of energy-rich or sugar-rich products.

In the USA, the National Weight Control Registry was established in 1993 to study formerly obese individuals who successfully lost a significant proportion of their obese body weight (10\% or more) and maintained a normal BMI for at least 5 years [22]. These "successful losers" share a number of behavioral characteristics, in comparison with persons who were never obese: they consume a low-fat diet, ingest less energy daily, have higher levels of physical activity, and consume more LES "light" beverages [23]. In this population, the regular intake of LES is associated with no sign of increased appetite in general or specific appetite for sweet-tasting products.

\section{Laboratory Studies}

A sensory evaluation test examined the taste responses to a sweetened fruit juice in 64 normal weight or overweight women [24]. Among the subjects, the participants who were frequent users of LES showed the same level of appreciation of 
the juice as women who were frequent users of sugar, again suggesting no heightened appetite for sweetness in users of LES.

In a laboratory study, eight habitual normal weight/ overweight consumers of large quantities of LES beverages (over $825 \mathrm{ml} / \mathrm{d}$ ) were compared to eight non-consumers [25]. The appetite for sweet or for savory foods was rated using visual analogue scales in the hours following the intake of either a sweet tasting or a non-sweet tasting lunch. A similar decrease in appetite for something sweet appeared in all groups following the sweet lunch. By contrast, an increase in subjective appetite for something sweet was noted in consumers of LES beverages following the nonsweet lunch, but not in non-consumers of LES. In spite of this difference in ratings of appetite, the actual intake at the next eating occasion was not different. So in this small group $(n=8)$ of very large consumers of LES, a significantly higher appetite for sweetness was observed in one experimental situation (following a nonsweet lunch) and was limited to subjective ratings but did not affect consumption. This study does not identify the causal links between reported observations. It remains unclear whether a high regular intake of LES might have modified appetite in consumers or whether individuals with a pre-existing high appetite for sweetness (possibly determined by a variety of reasons from genetics to culture and personal food history) were more likely to both become high users of LES and to demonstrate enhanced appetite for something sweet following a nonsweet tasting meal.

\section{Randomized Controlled Trials}

It is often suggested, as in the PNNS website for example [8], that the use of LES might prevent a "weaning" from sweet taste. This notion suggests, in particular, that dieters who limit their intake of sugar-containing products and do not use LES should experience a spontaneous decrease of liking for sweet products, as their diet would induce them to select healthier, non-sweet foods. Hypothetically, such a beneficial and spontaneous change might be antagonized by LES. In order to test this hypothesis, it should first be established that a decline in appetite for sweetness is actually induced by weight loss programs. Existing evidence does not support this notion. Quite the contrary, weight loss can induce an increase in taste sensitivity (lower perception thresholds) and liking for sweetness, as a result of changes in the hormonal status (decrease in leptin levels and increase in ghrelin [26, 27] among other effects). Diet-induced weight loss can therefore be accompanied by an increase in appetite for sweet tasting products whether or not LES are consumed during the diet. This effect of weight lossassociated hormonal changes might be incorrectly attributed to the use of LES.
The question of appetite for sweetness in dieters was addressed in a recently published RCT carried out in obese adults. In a weight loss intervention, participants $(n=104)$ were asked to replace daily intake of sugar-sweetened beverages with LES beverages, while another group $(n=106)$ was asked to replace sugar-sweetened beverages with water [28•]. The six-month intervention did not include any other dietary prescription (no caloric restriction). The hypothesis tested was that consumption of LES in beverages would enhance the consumption of sweet-tasting foods and beverages. Although weight loss was similar in both groups at the end of treatment (2-2.5\% of initial weight), several differences appeared over the intervention period between the water and LCS groups. In response to the intervention requirements, intake of diet beverages increased in the LES group and average daily intakes reached about 1 liter a day, in contrast to the intake observed in the water group (about $80 \mathrm{ml}$ a day at the end of the intervention). Therefore there is no question that the LES group was exposed to a high level of daily intake, likely capable of inducing the hypothesized increase in appetite for sweets.

The dietary changes recorded in the intervention group did not support the hypothesis. The participants exposed to a high level of intake of LES beverages for six months significantly reduced their sugar intake during the intervention. Notably, intake of sugar-containing beverages, including tea and coffee, dropped significantly as it did in the water group. In the LES beverage group, however, intake of desserts and caloric sweeteners decreased more than it did in the water group. In this RCT, spontaneous intake data brings no support to the notion that LES in beverages exert an enhancing effect on appetite in general, and on appetite for sweet-tasting products in particular. Actually the results suggested a broader suppression of appetite for sweetness in participants with a high daily intake of LES drinks than in the water group.

A recent report [29] of a 12-wk randomized behavioral intervention for weight loss indicates a higher weight loss in a group of obese participants required to drink LES beverages every day, compared to participants who were requested to drink equivalent amounts of water (5.95 versus $4.09 \mathrm{~kg}$ ). The larger weight loss in the LES group was accompanied by a larger reduction in subjective feelings of hunger than was observed in the water group. In fact, while the water group reported a small (non-significant) increase in experienced hunger from baseline to the end of intervention, the LES group reported a small (non-significant) decrease in experienced hunger. These divergent changes add up to produce a significant overall effect of treatments on hunger sensations at the end of the intervention. The specific appetite for sweetness or sweet-tasting products was not tested in this study. A 9-mo follow-up of this intervention is currently being carried out and will reveal the longer-term effects of the LES beverage treatment. 
The DRINK study is a randomized trial carried out in school children $[30,31,32 \bullet]$. In a sensory evaluation pre-test, it was shown that sugar-sweetened and LES beverages could be discriminated by taste but had the same palatability value. During an 18-month intervention 641 mostly normal weight children were randomized into two groups. One group received and drank a sugar-containing beverage (104 kcal) every day, whereas the control group received and drank a placebo drink (sweetened with LES). Over the intervention phase, no "compensation" appeared for the absence of energy from the LES drink, and experienced satiety was the same in both groups. Nevertheless, replacing sugar-containing beverages with sugar-free beverages reduced weight gain and body fat accumulation over the intervention period. Again this randomized controlled trial in children shows no support for the hypothesis that LES might exacerbate liking or wanting of sweet tasting products.

\section{Functional Magnetic Resonance Imaging Studies}

The recent development of brain activation studies using functional magnetic resonance imaging (fMRI) has established that the human brain can dissociate a sweet nutritive taste from a non-nutritive one [33-35]. A few studies reported differences in the brain responses to sweetness in regular users of LES versus non-users. A negative association between the use of LES and the reward-signaling amygdala response to sucrose ingestion was observed in one study of normal weight/ overweight persons [36]. By contrast, another work reported that normal weight/overweight individuals with frequent intake of LES soda exhibited greater activation in response to sweet taste (sucrose and saccharin) in the dopaminergic midbrain and right amygdala as well as in reward processing brain regions such as the orbitofrontal cortex [37]. Methodological differences could perhaps account for these apparently discrepant reports.

Although future fMRI studies will certainly bring more evidence on brain responses associated with regular use of LES, it is important to remember that cross-sectional studies, such as the fMRI works published so far, cannot possibly demonstrate causal relationships, whatever their level of technical sophistication. Observing that some brain regions are more or less activated by sweet tasting stimuli of various kinds does not inform about the causal mechanisms involved. It is possible that the frequent intake of LES in foods and beverages might increase the liking for such products, thereby inducing changes in brain responses; conversely, it is possible that the observed differences in brain activity might reflect initially higher liking or wanting for sweet tasting products and/or difficulties in appetite control that might lead individuals to adopt LES. More research is needed before we can make sense of the results of fMRI studies and interpret the critical causal relationships.

\section{Conclusions}

LES are a class of substances that share an intense sweet taste but vary considerably in their physicochemical structures. Their safety is under continuous scrutiny by public health authorities in the developed world. For instance, the safety of one LES, aspartame, was re-affirmed by the European Food Safety Authority (EFSA) in December 2013 following an extended review of all relevant literature [38]. While the concerns for safety will continue to be monitored by the relevant public organizations, the conditions of use for optimal benefits have to be identified.

Overall, the existing studies, using widely differing methodologies in various types of consumers (men, women, lean, obese, never obese, formerly obese), reach largely convergent conclusions: the use of LES shows no consistent association with a heightened appetite for sugar or sweet products. In fact, in many instances, the use of LES is associated with a lower intake of sweet tasting substances. Recent intervention studies in children and adults confirm that LES use tends to reduce rather than increase the intake of sugar-containing foods, and to facilitate, rather than impair, weight loss. Longer-term randomized studies are needed to confirm the benefits of LES in different aspects of weight control: prevention of weight gain, weight loss, and/or maintenance of weight loss.

\section{Compliance with Ethics Guidelines}

Conflict of Interest The present review is original and has never been published elsewhere. However, several parts of it are extracted from a non-published document for which the author received an honorarium from the International Sweeteners Association (ISA).

France Bellisle is a member of the Scientific Advisory Board for General Mills and has received travel reimbursement and honoraria for contributions in scientific congresses from Mondelez, ISA, and General Mills.

Human and Animal Rights and Informed Consent This article does not contain any studies with human or animal subjects performed by the author.

\section{References}

Papers of particular interest, published recently, have been highlighted as:

- Of importance

1. Fernstrom JD, Munger SD, Sclafani A, de Arujo I, Roberts A, Molinary S. Mechanisms for sweetness -low-calorie sweeteners, appetite and weight control: what the science tells us. J Nutr. 2012;142:1134S.

2. Erlanson-Albertsson C. How palatable food disrupts appetite regulation. Basic Clin Pharmacol Toxicol. 2005;97:61-73. 
3. Ifland JR, Preuss HG, Marcus MT, Rourke KM, Taylor WC, Burau $\mathrm{K}$, et al. Refined food addiction: a classic substance use disorder. Med Hypotheses. 2009;72:518-26.

4. Fowler SP, Williams K, Resendez RG, Hunt KJ, Hazuda HP, Stern MP. Fueling the obesity opidemic? Artificially sweetened beverage use and long-term weight gain. Obesity. 2008;16:1894-900.

5. Pereira MA. Diet beverages and the risk of obesity, diabetes, and cardiovascular disease: a review of the evidence. Nutr Rev. 2013;71:433-40

6. Swithers SE. Articifical sweeteners product the counterintuitive effect of inducing metabolic derangements. Trends Endocrinol Metab. 2013;24:431-41.

7. Mattes RD, Popkin BM. Nonnutritive sweetener consumption in humans: effects on appetite and food intake and their putative mechanisms. Am J Clin Nutr. 2009;89:1-14.

8. Plan National Nutrition Santé (2002) Paris: Agence Française pour la Sécurité Alimentaire. http://www.mangerbouger.fr/bien-manger/ que-veut-dire-bien-manger-127/les-9-reperes/produits-sucres-alimiter.html.

9. Avena NM, Rada P, Hoebel BG. Evidence for sugar addiction: behavioral and neurochemical effects of intermittent, excessive sugar intake. Neurosci Biobehav Rev. 2008;32:20-39.

10. Benton $\mathrm{D}$. The plausibility of sugar addiction and its role in obesity and eating disorders. Clin Nutr. 2010;29:288-303.

11. Blundell JE, Hill AJ. Paradoxical effects of an intense sweetener (aspartame) on appetite. Lancet. 1986;10:1092-3.

12. Blundell JE, Rogers PJ, Hill AJ. Uncoupling sweetness and calories: Methodological aspects of laboratory studies on appetite control. Appetite. 1988;11(suppl):54-61.

13. Rogers PJ, Blundell JE. Separating the actions of sweetness and calories: Effects of saccharin and carbohydrates on hunger and food intake in human subjects. Physiol Behav. 1989;45:1093-9.

14. Rogers PJ, Blundell JE. Intense sweeteners and appetite. Am J Clin Nutr. 1993;58:120-1.

15. Rogers PJ, Carlyle JA, Hill AH, Blundell JE. Uncoupling sweet taste and calories: Comparison of the effects of glucose and three intense sweeteners on hunger and food intake. Physiol Behav. 1988;43:547-52.

16. Cabanac M, Duclaux R. Alliesthesie olfactory-gustative et prise alimentaire chez l'homme. J Physiologie (Paris). 1973;66:113-35.

17. De la Hunty A, Gibson S, Ashwell M. A review of the effectiveness of aspartame in helping with weight control. Nutr Bull. 2006;31:115-28.

18. Miller PE, Perez V. Low-calorie sweeteners and body weight and composition: a meta-analysis of randomized controlled trials and prospective cohort studies. Am J Clin Nutr. 2014. doi:10.3945/ajen.113.082826.

19. Hercberg S, Preziosi P, Briançon S, Galan P, Triol I, Malvy D, et al. A primary prevention trial using nutritional doses of antioxidant vitamins and minerals in cardiovascular disease and cancers in a general population: the SU.VI.MAX study - design, methods, and participants characteristics. Control Clin Trials. 1998;19:336-51.

20. Bellisle F, Altenburg de Assis MA, Fieux B, Preziosi P, Galan P, Guy-Grand B, et al. The use of light foods and drinks in French adults: biological, anthropometric and nutritional correlates. J Hum Nutr Diet. 2001;18:197-205.

21. Binkley J, Golub A. Comparison of grocery purchase patterns of diet soda buyers to those of regular soda buyers. Appetite. 2007;49: 561-71.

22. Klem ML, Wing RR, McGuire MT, Seagle HM, Hill JO. A descriptive study of individuals successful at long-term maintenance of substantial weight loss. Am J Clin Nutr. 1997;66:239-46.
23. Phelan S, Lang W, Jordan D, Wing RR. Use of artificial sweeteners and fat-modified foods in weight loss maintainers and always normal weight individuals. Int J Obes. 2009;33: 1183-90.

24. Mahar A, Duizer LM. The effect of frequency of consumption of artificial sweeteners on sweetness liking by women. J Food Sci. 2007;72:S714-8.

25. Appleton KM, Rogers PJ, Blundell JE. Effects of a sweet and a nonsweet lunch on short-term appetite: differences in female high and low consumers of sweet/low-energy beverages. J Hum Nutr Diet. 2004; 17:425-34.

26. Disse E, Bussier AL, Veyrat-Durebex C, Deblon N, Pfluger PT, Tschop MH, et al. Peripheral ghrelin enhances sweet taste food consumption and preference, regardless of its caloric content. Physiol Behav. 2010;101:277-81.

27. Umakiki M, Tsuzaki K, Kotani K, Nagai N, Sano Y, Matsuoda Y, et al. The improvement of sweet taste sensitivity with decrease in serum leptin levels during weight loss in obese females. Tohoku $\mathrm{J}$ Exp Med. 2010;222:267-71.

28. Piernas C, Tate DF, Wang X, Popkin BM. Does diet-beverage intake affect dietary consumption patterns? Results from the Choose Healthy Options Consciously Everyday (CHOICE) randomized clinical trial. Am J Clin Nutr. 2013;97:604-11. RCT in adults showing a spontaneous decrease in intake of sugar and sweet foods and drinks in LES intervention group.

29. Peters JC, Wyatt HR, Foster GD, Pan A, Wojtanowski AC, Vander Veur SS, et al. The effects of water and non-nutritive sweetened beverages on weight loss during a 12-week weight loss treatment program. Obesity. 2014;22:1415-21.

30. De Ruyter J, Olthof MR, Seidell JC, Katan MB. A trial of sugar-free or sugar-sweetened beverages and body weight in children. N Engl J Med. 2012;367:1397-406.

31. De Ruyter J. A double-blind randomized trial in school-children on the effects of sugar-sweetened or sugar-free beverages on body weight and body fatness. Doctoral Dissertation, University of Amsterdam, 2013.

32. De Ruyter JC, Katan MB, Kuijper LDJ, Liem DG, Olthof MR. The effect of sugar-free versus sugar-sweetened beverages on satiety, liking and wanting: An 18 month randomized double-blind trial in children. Plos One. 2013;8:e78039. RCT in children showing beneficial effects on weight change without any increase in appetite in LES intervention group.

33. Frank GK, Oberndorfer TA, Simmons AN, Paulus MP, Fudge JL, Yang TT, et al. Sucrose activates human taste pathways differently from artificial sweetener. Neuroimage. 2008;39:1559-69.

34. Chambers ES, Bridge MW, Jones DA. Carbohydrate sensing in the human mouth: effects on exercise performance and brain activity. $\mathrm{J}$ Physiol. 2009;587:1779-94.

35. Smeets PA, Weizen P, de Graaf C, Viergever MA. Consumption of caloric and non-caloric versions of a soft drink differentially affects brain activation during tasting. Neuroimage. 2010;54:1367-74.

36. Rudenga KJ, Small DM. Amygdala response to sucrose consumption is inversely related to artificial sweetener use. Appetite. 2012;58:504-7.

37. Green E, Murphy C. Altered processing of sweet taste in the brain of diet soda drinkers. Physiol Behav. 2012;107:560-7.

38. EFSA. Panel of food additives and nutrient sources added to foods (ANS). Endorsed for public consultation. Draft scientific opinion on the reevaluation of aspartame (E951) as a food additive, 1-245, January 8 (2013). http://www.efsa.eu. 